\title{
An Empirical Path-Loss Model for Wireless Channels in Indoor Short-Range Office Environment
}

\author{
Ye Wang, Wen-jun Lu, and Hong-bo Zhu \\ Jiangsu Key Laboratory of Wireless Communications, College of Telecommunications and Information Engineering, \\ Nanjing University of Posts \& Telecommunications, Nanjing 210003, China \\ Correspondence should be addressed to Hong-bo Zhu, wilab@njupt.edu.cn
}

Received 31 August 2011; Accepted 3 November 2011

Academic Editor: Dau-Chyrh Chang

Copyright ( 2012 Ye Wang et al. This is an open access article distributed under the Creative Commons Attribution License, which permits unrestricted use, distribution, and reproduction in any medium, provided the original work is properly cited.

A novel empirical path-loss model for wireless indoor short-range office environment at $4.3-7.3 \mathrm{GHz}$ band is presented. The model is developed based on the experimental datum sampled in 30 office rooms in both line of sight (LOS) and non-LOS (NLOS) scenarios. The model is characterized as the path loss to distance with a Gaussian random variable $X$ due to the shadow fading by using linear regression. The path-loss exponent $n$ is fitted by the frequency using power function and modeled as a frequencydependent Gaussian variable as the standard deviation $\sigma$ of $X$. The presented works should be available for the research of wireless channel characteristics under universal indoor short-distance environments in the Internet of Things (IOT).

\section{Introduction}

The Internet of Things that preliminary conception in the International Telecommunications Union (ITU) Internet Report (series 7) in 1997 is constructing a network what is covering everything of the world. In November 2005, the ITU provided a perfect and succinct definition [1]: from anytime, anyplace connectivity for anyone, we will now have connectivity for anything. Connections will multiply and create an entirely new dynamic network of networks. The network is a ubiquitous network, meeting with the general ubiquitous system-the Internet of Things (IOT). In the future, the IOT should be a ubiquitous network what all kinds of network and "things" need to merge in. Thus, it is essential to develop an accurate path-loss model for the propagation characteristics of different frequency bands for the path-loss model generally determines the requirements for power control, sensor node spacing, radio-frequency identification (RFID) design, and so on.

Many propagation characteristics have been extensively studied for indoor channels over the past two decades [2$7]$, and several models have focused on specific indoor environments [8-13]. In this paper, the large-scale propagation characteristics of wireless channels under indoor shortdistance office environment have been researched. Based on the experimental results, a novel empirical path-loss model with two important parameters ( Tx-Rx distance and carrier frequency) is proposed by using linear regression. Specifically, the path-loss model describes the statistical characteristics for parameters. And simulations were conducted to verify the model.

\section{Measurement Procedure}

2.1. Equipment and Measurement System. The diagram of the measurement system used for frequency-domain characterization of indoor short-range wireless channel is shown in Figure 1. A vector network analyzer (VNA, e.g., HP8720ES) is used to sweep 201 continuous wave tones uniformly distributed from 4.3 to $7.3 \mathrm{GHz}$, with a frequency separation of $15 \mathrm{MHz}$. The signal generated by the VNA is as the input to a variable attenuator and a transmitter RF amplifier with a gain of 33-dB. The output of the RF amplifier is propagated by a vertical-polarized, omnidirectional, diamond-like disc monopole antenna with a gain of $0 \mathrm{dBi}$. The signal from the identical receive antenna is first passed through a low-noise amplifier (LNA) with a gain of $32 \mathrm{~dB}$. It is then transmitted via 30 meters coaxial cable with a $15-\mathrm{dB}$ loss followed by a RF amplifier with a gain of $36-d B$. Finally, the output 


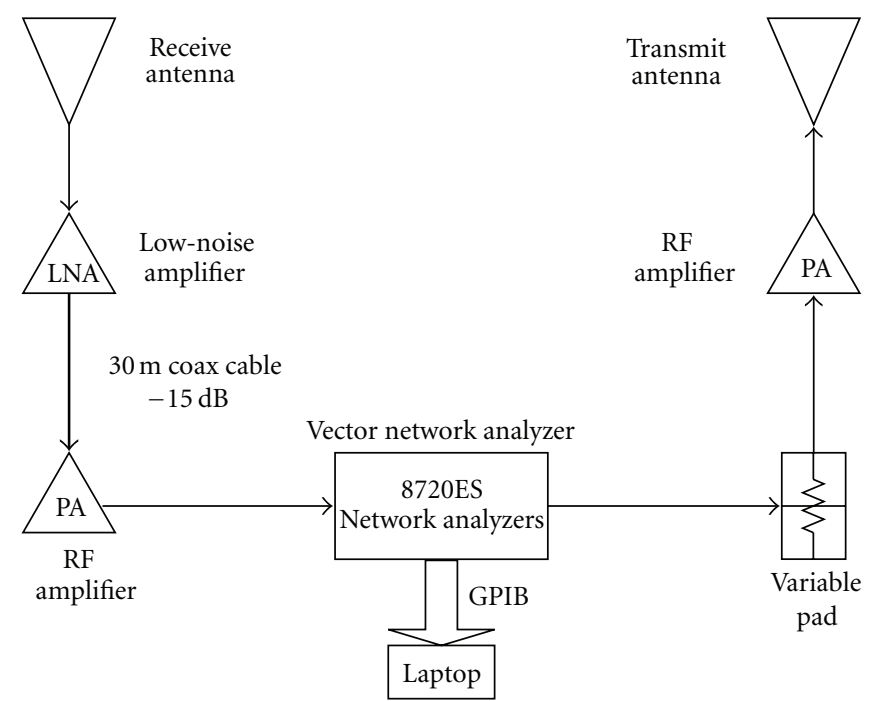

FIGURE 1: Block diagram of the measurement system.

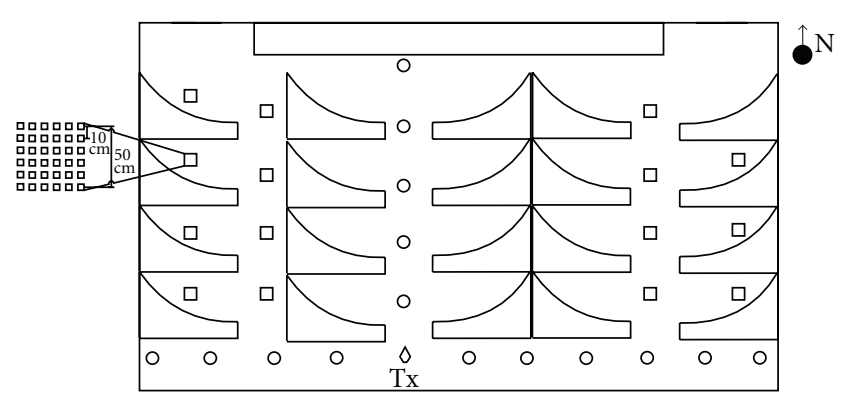

$\square$ NLOS location

- LOS location

Figure 2: Illustration of the measurement locations in a typical office room.

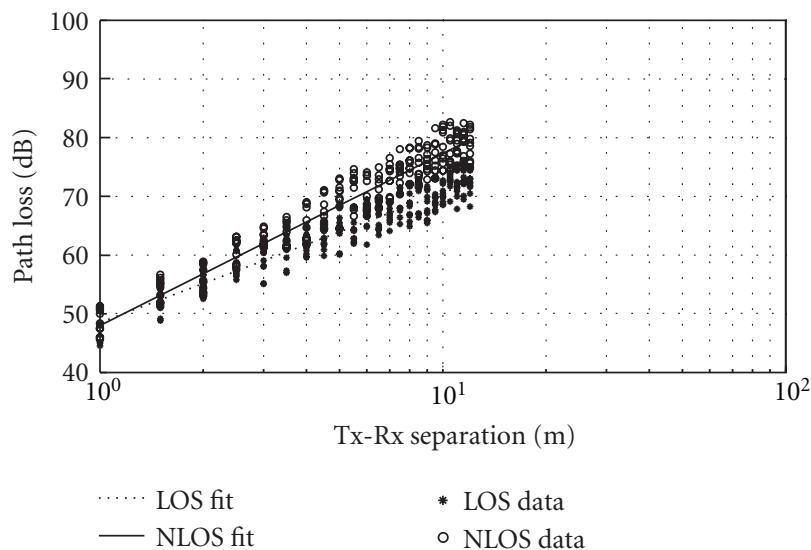

Figure 3: Scatter plots of path loss versus T-R separation in shortrange office environment at $5.8 \mathrm{GHz}$. The straight line is least squares linear regression fit.

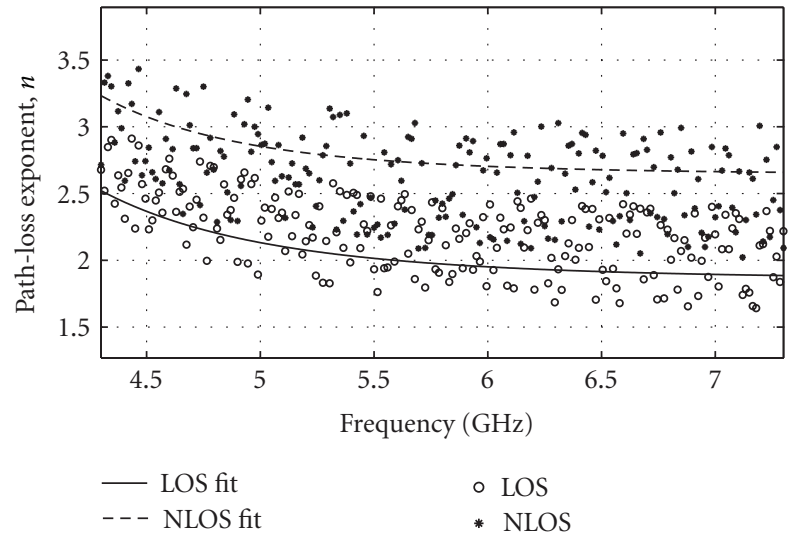

FIGURE 4: Scatter plots of path-loss exponent $n$ and corresponding frequency. The curves are least squares regression fit to the formula $n=a_{1} f^{a_{2}}+a_{3}$.

of the amplifier returned to the VNA. The time to sweep the frequency band is $400 \mathrm{~ms}$. In addition, the intermediate frequency bandwidth is $3000 \mathrm{~Hz}$. The measured complex frequency response is stored on a computer hard drive via a GPIB interface controlled by Agilent-VEE programs.

This setup was properly calibrated in an anechoic chamber to compensate for the effects of impedance mismatching of the antennas with the front end of the transceiver. The calibration data removed the effects of hardware and was also for postprocessing and reduction of data.

2.2. Measurement Environment and Database Collected. Measurements were carried out inside 30 office rooms in Nanjing and Beijing. The office rooms had been differing on structure, age, layout, and size. However, the sizes of office rooms are all within 15 by $15 \mathrm{~m}^{2}$ under a short-range environment. 


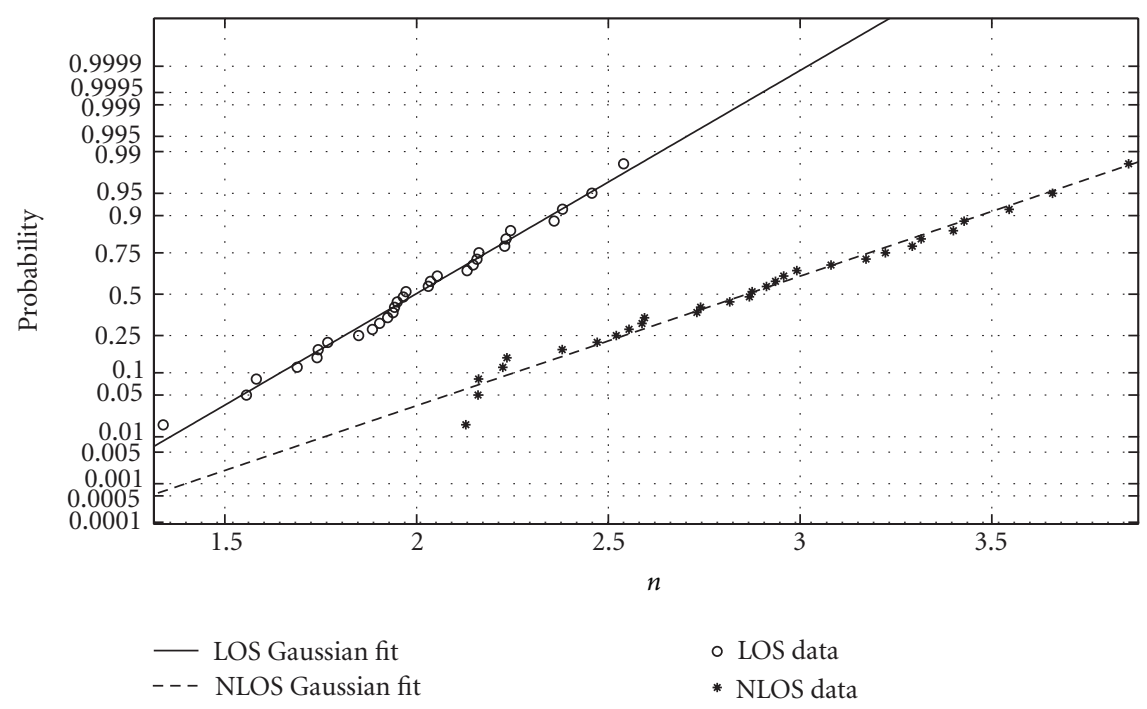

FIGURE 5: CDF of the deviation of path-loss exponent $n$ in all measurement office rooms at $5.8 \mathrm{GHz}$.

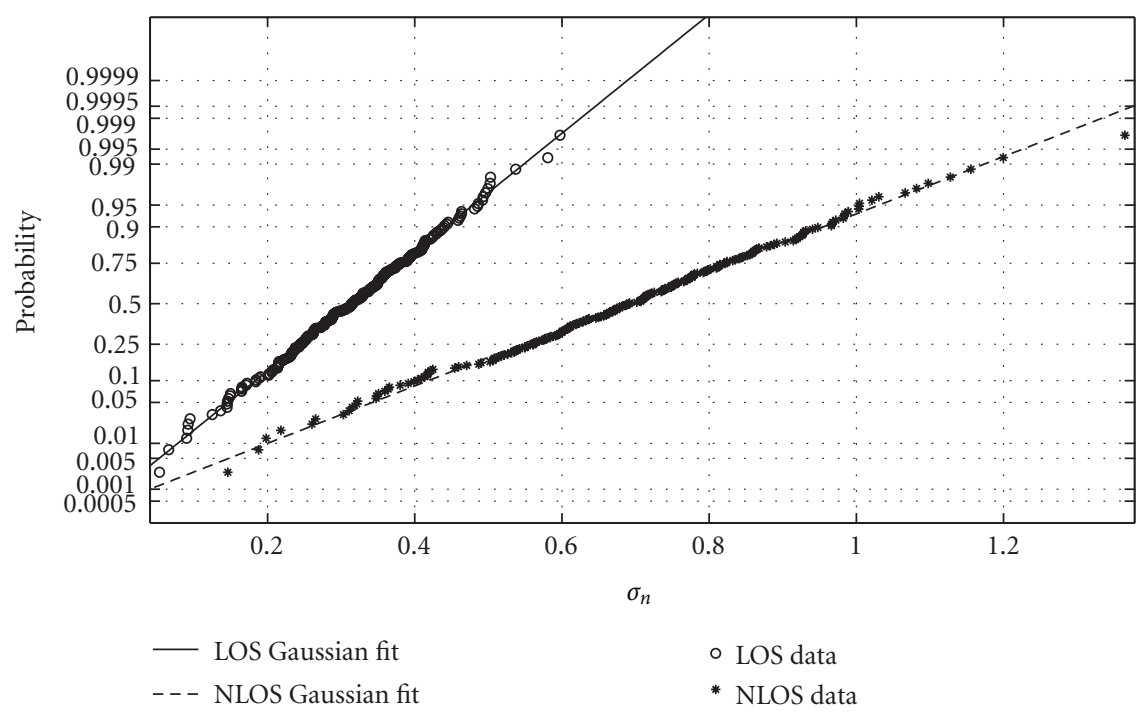

FIgURE 6: CDF of the standard deviation of path-loss exponent $\sigma_{n}$ over 201 frequency points.

In each office room, 15 locations were selected for lineof-sight (LOS) and non-line-of-sight (NLOS) scenarios, respectively. In NLOS scenario, the waves were obstructed by the tables partitioning by over $1.5 \mathrm{~m}$ height partition or furnishings in the rooms as the typical office room shown in Figure 2. The transmitter-receiver (T-R) separation, $d$, is ranging from $1 \mathrm{~m}$ to $12 \mathrm{~m}$. In each room, the transmit antenna was keeping fixed in a given position which is the best place for signal coverage in the office room. The receive antenna was moved throughout the preprepared locations. In each location, measurements were made at 36 points, arranged in a $6 * 6$ square grid with $10-\mathrm{cm}$ spacing, covering $50 \mathrm{~cm} * 50 \mathrm{~cm}$. It is also shown in Figure 2. Transmit and receive antennas during all the measurements were in the same horizontal plane, while the height of antennas was $1.2 \mathrm{~m}$.

\section{Data Reductions and Analysis}

3.1. Data Reductions. Based on the stored calibration data, for each measurement location, the database consists of 36 channel complex frequency responses, $H_{k}\left(f_{i}\right)$. Here, the index $k$ denotes one of the 36 grid points and $f_{i}$ are 201 discrete frequencies ranging from 4.3 to $7.3 \mathrm{GHz}$ (i.e., $1 \leq$ $k \leq 36$ and $0 \leq i \leq 200$ ). The mean path loss (in $\mathrm{dB}$ ) in a measurement location with T-R separation $d$ at the frequency $f_{i}$ is estimated as the spatial average of 36 grid points, by

$$
\operatorname{PL}\left(d, f_{i}\right)=10 \log _{10} \frac{1}{36} \sum_{k=1}^{36}\left|H_{k}\left(f_{i} ; d\right)\right|^{2} .
$$




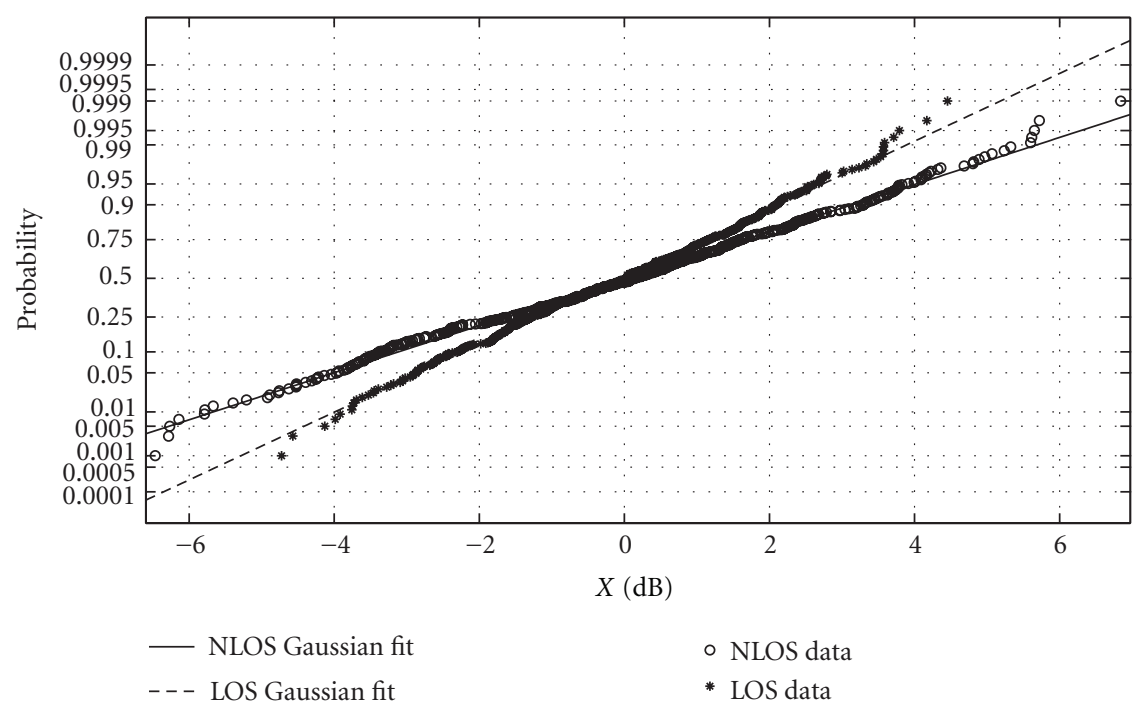

Figure 7: CDF of the shadow fading component $X$ in a typical office room at $5.8 \mathrm{GHz}$. This case is representative of $201 \mathrm{frequency}$ points in the database.

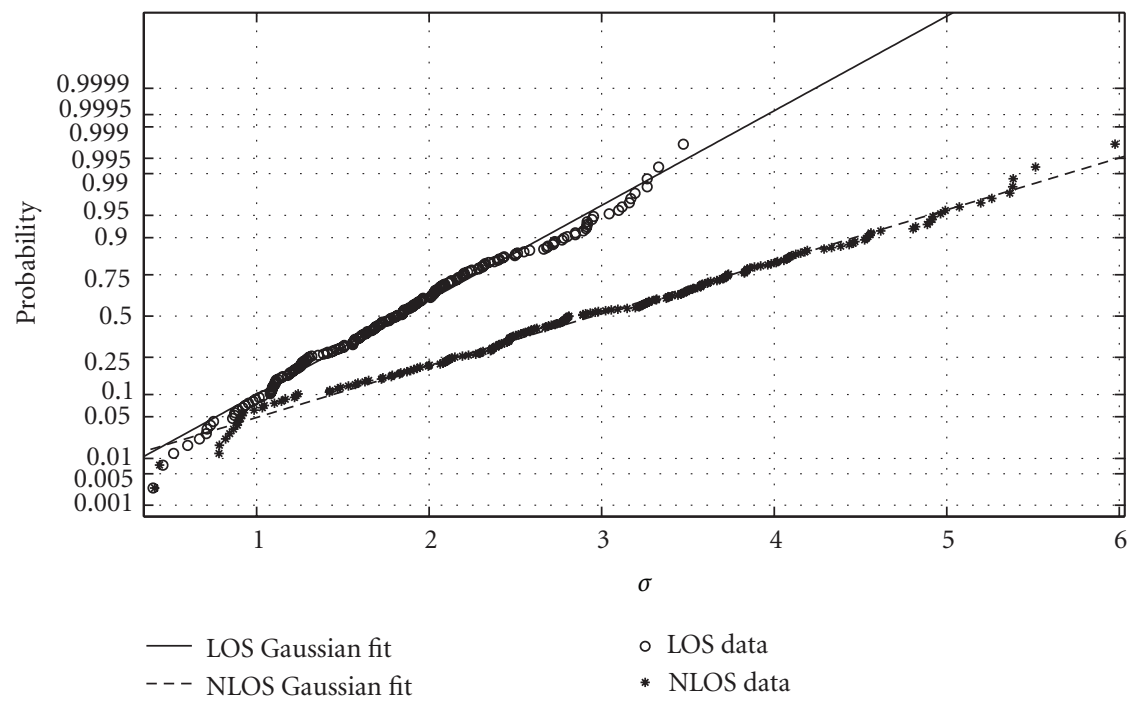

Figure 8: CDF of the shadow fading standard deviation $\sigma$ from 201 frequency points.

3.2. Data Analysis. A general path-loss expression that includes reflection, diffraction, and scattering for both LOS and NLOS scenarios is usually to represent path loss by using a path-loss exponent $n$, plus a random variation due to the shadowing effects. Thus, the path-loss model for a T-R separation $d$ at the frequency $f$ is given as

$$
\begin{array}{r}
\mathrm{PL}(d, f)=\operatorname{PL}\left(d_{0}\right)+10 n \log _{10}\left(\frac{d}{d_{0}}\right)+X, \quad d \geq d_{0} \\
\text { (antenna gain included), }
\end{array}
$$

where $\operatorname{PL}\left(d_{0}\right)$ is the decibel path loss at close-in distance $d_{0}$ and $X$ is the shadow fading. $d_{0}$ is chosen a value of $1 \mathrm{~m}$ and $\sigma$ is minimized in each frequency point through measurement data.

The scatter plots of path loss computed by (1) in locations of all office rooms for LOS and NLOS scenarios at
5.8 GHz are depicted in Figure 3. The lines are least squares linear regression lines through the scatter of path loss in locations estimated by (2). The root mean square deviation of path-loss points about the regression line is minimized.

Random shadowing effects of the channel, $X$, occur at locations where the T-R separation is the same but have different levels of clutter in their propagation paths. $X$ is usually a zero mean normal random variable with standard deviation $\sigma$ (dB units).

\section{Key Findings}

In fact, in [14], a statistical path-loss model had been created by taking $n$ and $\sigma$ as random variables over buildings.

This follows a similar approach used for path-loss modeling in large outdoor cells [15]. In office rooms under 


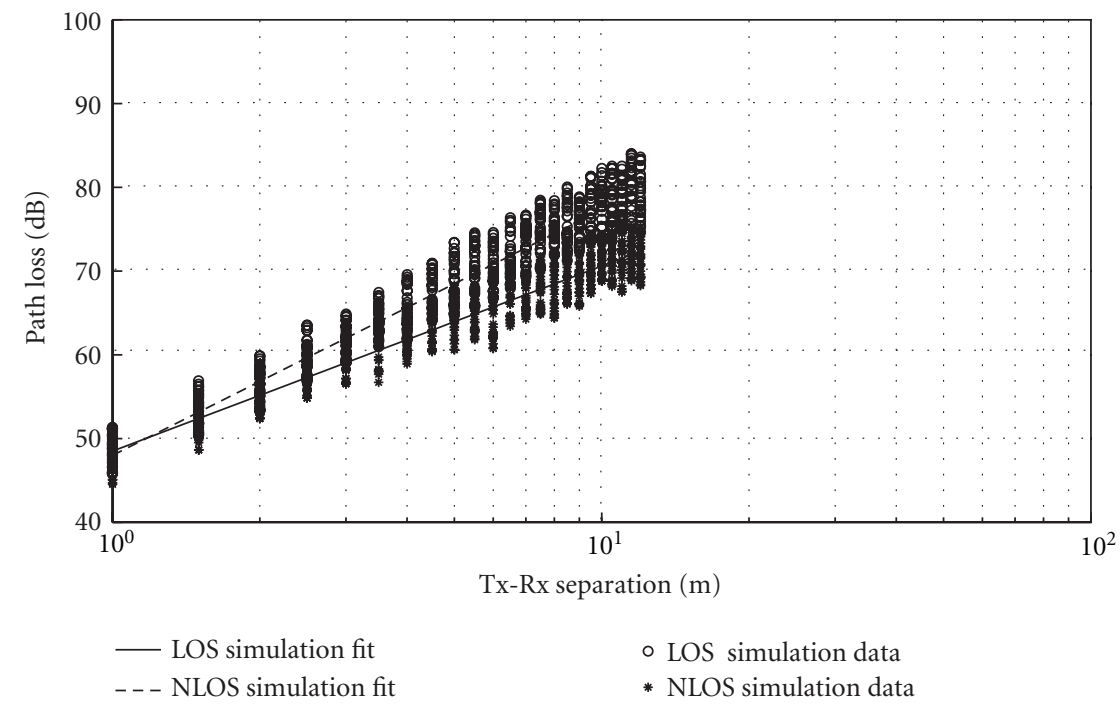

FIGURE 9: Scatter plots of simulation results at $5.8 \mathrm{GHz}$.

the short-range environment, by characterizing the model parameters over all 201 frequency points, the following key findings were found.

The values of $\operatorname{PL}\left(d_{0}\right)$ at the frequency $f$ were measured in LOS scenario for both LOS and NLOS scenarios, and the values were within few decibels of the path-loss value of $1 \mathrm{~m}$ in free space. Thus, $\operatorname{PL}\left(d_{0}\right)$ may be modeled by the formula $20 \log _{10}\left(4 \pi d_{0} / \lambda\right)$ for all 201 frequency points as in free space, where $\lambda=C / f$ is wavelength in meters. $C$ is the speed of light.

In Figure 4, scatter plots of $n$ and frequency are depicted for both LOS and NLOS. It is seen that $n$ is slight frequency dependent under such a short-range office propagation environment. As shown in Figure 4, higher frequency leads to smaller $n$. The curves in Figure 4 are fitted with the function $n=a_{1} f^{a_{2}}+a_{3}$ for LOS and NLOS, where $f$ is in GHz. Each $n$ value was calculated under each $\operatorname{PL}\left(d_{0}\right)$ at the corresponding frequency $f$.

The path-loss exponent $n$ at each frequency point is indeed changed from one office room to another and has an approximate Gaussian distribution. The distribution of $n$ at $5.8 \mathrm{GHz}$ for LOS and NLOS is illustrated in Figure 5, where the straight lines mean Gaussian distribution. Further, over the analysis of all 201 frequency points, the standard deviation of $n$ (indicated by $\sigma_{n}$ ) is shown to be an approximate Gaussian distribution too. The distribution of $\sigma_{n}$ is shown in Figure 6, where the straight lines mean Gaussian distribution.

In Figure 7, the distribution of shadow fading $X$ in a typical office room at $5.8 \mathrm{GHz}$ is depicted. The normal distribution regression line fit to the $\mathrm{dB}$ values confirmed the log-normality of shadow fading. $\sigma$, the standard deviation of $X$, is a random variable from one frequency to another, and it is generally Gaussian distribution in measure frequency points. The distribution of $\sigma$ in all 201 frequency points is shown in Figure 8.

\section{Path-Loss Model and Simulations}

5.1. The Path-Loss Model. Based on the above discussions, the path-loss model for indoor short-range office environment is constructed. The model is derived from the frequency response datum sampled at frequency range from 4.3 to $7.3 \mathrm{GHz}$ in 30 office rooms under short-range environment.

The $\operatorname{PL}\left(d_{0}\right)$ is a fixed quantity at each frequency and is given as (3) for LOS and NLOS scenarios:

$$
\mathrm{PL}\left(d_{0}\right)=20 \log _{10}\left(\frac{4 \pi d_{0}}{\lambda}\right)=20 \log _{10}\left[\frac{4 \pi d_{0}}{(C / f)}\right],
$$

where $d_{0}=1 \mathrm{~m}, \lambda$ is the wavelength, unit in meter, and $C$ is the speed of light.

The path-loss exponent $n$ is a Gaussian random variable described as

$$
n=\left[a_{1} f^{a_{2}}+a_{3}\right]+z_{1} \sigma_{n}, \quad 4.3 \mathrm{GHz} \leq f \leq 7.3 \mathrm{GHz} .
$$

The part in brackets gives the mean value of $n\left(a_{1}, a_{2}, a_{3}\right.$ in unity units and $f$ in $\mathrm{GHz}$ ). The standard deviation $\sigma_{n}$ of $n$ is also a Gaussian random variable described as

$$
\sigma_{n}=\mu_{\sigma_{n}}+z_{2} \sigma_{\sigma_{n}}
$$

The shadow fading $X$ is a zero-mean normal variable, denoted as

$$
X=0+z_{3} \sigma=z_{3} \sigma .
$$

$\sigma$, the standard deviation of $X$, is also a Gaussian variable over all test frequency points. So it is shown as

$$
\sigma=\mu_{\sigma}+z_{4} \sigma_{\sigma}
$$

where $\mu_{\sigma}$ is the mean of $\sigma ; \sigma_{\sigma}$ is the standard deviation of $\sigma ; z_{1}, z_{2}, z_{3}$, and $z_{4}$ mentioned above are zero-mean Gaussian 
TABLE 1: Values of model parameters.

\begin{tabular}{lcc}
\hline & LOS & NLOS \\
\hline$a_{1}$ & 3176 & 12160 \\
$a_{2}$ & -5.8 & -6.8 \\
$a_{3}$ & 1.8 & 2.6 \\
$\mu_{\sigma_{n}}$ & 0.31 & 0.72 \\
$\sigma_{\sigma_{n}}$ & 0.10 & 0.28 \\
$\mu_{\sigma}$ & 1.8 & 3.0 \\
$\sigma_{\sigma}$ & 0.7 & 1.2 \\
\hline
\end{tabular}

TABle 2: Parameter values of measurement and simulation at $5.8 \mathrm{GHz}$.

\begin{tabular}{lccc}
\hline & $\operatorname{PL}\left(d_{0}\right)$ & $n$ & $\sigma$ \\
\hline LOS measurement & $48.2 \mathrm{~dB}$ & 2.06 & 1.92 \\
NLOS measurement & $48.5 \mathrm{~dB}$ & 2.84 & 3.20 \\
LOS simulation & $48.1 \mathrm{~dB}$ & 2.01 & 2.01 \\
NLOS simulation & $48.7 \mathrm{~dB}$ & 2.77 & 3.12 \\
\hline
\end{tabular}

variables of unit standard deviation, $N[0,1]$. The numerical values of these constants are all given in Table 1 .

Combining the above analysis, the path-loss model is given as

$$
\begin{aligned}
\mathrm{PL}= & {\left[20 \log _{10}\left(\frac{4 \pi d_{0}}{(C / f)}\right)+10\left(a_{1} f^{a_{2}}+a_{3}\right) \log _{10}\left(\frac{d}{d_{0}}\right)\right] } \\
& +\left[\left(10 z_{1} \mu_{\sigma_{n}}+10 z_{1} z_{2} \sigma_{\sigma_{n}}\right) \log _{10}\left(\frac{d}{d_{0}}\right)+z_{3} \mu_{\sigma}+z_{3} z_{4} \sigma_{\sigma}\right],
\end{aligned}
$$

where, in the first brackets, the median path loss at the separation of Tx-Rx $d$ and frequency $f$ is described and, in the second brackets, the random variation about the median is described. In the second brackets, $z_{1}$ varies from room to room, while $z_{2}$ and $z_{4}$ vary from frequency to frequency, and $z_{3}$ varies from location to location within each room.

5.2. The Simulations. Finally, in using this model for simulations, it would be prudent to use truncated Gaussian distributions for $z_{1}, z_{2}, z_{3}$, and $z_{4}$ so as to keep the measurement variables in appearing practicable values. One such possibility is to limit them to the following ranges:

$$
z_{1} \in[-0.5,0.5], \quad z_{2}, z_{4} \in[-1.5,1.5], \quad z_{3} \in[-1,1] .
$$

The results were simulated by path-loss model (8) and compared to the data obtained from measurements. We generated 30 realizations of $z_{1}, 25$ pairs of $d$ and $z_{3}$, and 201 pairs of $z_{2}$ and $z_{4}$. In Figure 9, the simulated results at $5.8 \mathrm{GHz}$ are shown compared to measured scatter plots in the Figure 3. Parameter values of measurement and simulation at 5.8 GHz are given in Table 2 .

\section{Conclusion}

In this paper, a novel statistical prediction path-loss model for short-distance indoor office environment at $4.3-7.3 \mathrm{GHz}$ is presented. A complete characterization of the model parameters was described, along with probability distributions and dependencies between parameters. The distinctions of the model among different frequencies are significant as experimental data shows the difference of path-loss value at different frequencies is obvious.

The above model shows equilibrium of including as many details as possible and minimizing the complexity. What is more, the new model explains the relationship between the path-loss exponent and the work frequency together produces a novel framework about describing the path loss. It provides a certain basis for the realization of wireless communication under complicated environment with complex utilizing of the spectrum resource.

Since the data are measured and analyzed only under office environment, some other complicated indoor environment will be studied in further such as commercial environment, residential environment, stair corner environment, and the long corridor environment. One hand, more precise statistical characteristics of $n$ and $\sigma$ about environment variability will improve and enrich the model via a larger database. On the other hand, channel digital transmission technology and performance will study synchronously.

\section{Acknowledgment}

This work was supported by National Science and Technology Major Project under Grant no. 2011ZX03005-004-03 and National Science and Technology Major Project under Grant no. 2012ZX03001028-005.

\section{References}

[1] International Telecommunication Union, ITU reports 2005 executive summary: Internet of Things, http://www.itu.int/ osg/spu/publications/internetofthings/InternetofThings_summary.pdf.

[2] A. A. M. Saleh and R. A. Valenzuela, "A statistical model for indoor multipath propagation," IEEE Journal on Selected Areas in Communications, vol. 5, no. 2, pp. 128-137, 1987.

[3] S. J. Howard and K. Pahlavan, "Measurement and analysis of the indoor radio channel in the frequency domain," IEEE Transactions on Instrumentation and Measurement, vol. 39, no. 5, pp. 751-755, 1990.

[4] H. Hashemi, "The indoor radio propagation channel," Proceedings of the IEEE, vol. 81, no. 7, pp. 943-968, 1993.

[5] D. Cassioli, M. Z. Win, and A. F. Molisch, "The ultra-wide bandwidth indoor channel: from statistical model to simulations," IEEE Journal on Selected Areas in Communications, vol. 20, no. 6, pp. 1247-1257, 2002.

[6] J. Foerster, Ed., "Channel Modeling Sub-committee Report Final," IEEE802.15-02/490, http://ieee802.org/15/.

[7] S. S. Ghassemzadeh, R. Jana, C. W. Rice, W. Turin, and V. Tarokh, "Measurement and modeling of an ultra-wide bandwidth indoor channel," IEEE Transactions on Communications, vol. 52, no. 10, pp. 1786-1796, 2004. 
[8] S. Y. Seidel and T. S. Rappaport, "914 MHz path loss prediction models for indoor wireless communications in multifloored buildings," IEEE Transactions on Antennas and Propagation, vol. 40, no. 2, pp. 207-217, 1992.

[9] J. Poutanen, K. Haneda, J. Salmi, V. M. Kolmonen, J. Koivunen, and P. Almers, "Analysis of radio wave propagation from an indoor hall to a corridor," in Proceedings of the IEEE Antennas and Propagation Society International Symposium and Usnc/Ursi National Radio Science Meeting, vol. 1-6, pp. 2683-2686, 2009.

[10] N. Noori, R. Karimzadeh-Baee, and A. Abolghasemi, "An empirical ultra wideband channel model for indoor laboratory environments," Radioengineering, vol. 18, no. 1, pp. 68-74, 2009.

[11] S. Geng and P. Vainikainen, "Millimeter-wave propagation in indoor corridors," IEEE Antennas and Wireless Propagation Letters, vol. 8, Article ID 5308233, pp. 1242-1245, 2009.

[12] S. Y. Lim, Z. Yun, J. M. Baker, N. Celik, H. S. Youn, and M. F. Iskander, "Propagation modeling and measurement for a multifloor stairwell," IEEE Antennas and Wireless Propagation Letters, vol. 8, Article ID 4907144, pp. 583-586, 2009.

[13] A. Valcarce and J. Zhang, "Empirical indoor-to-outdoor propagation model for residential areas at 0.9-3.5 GHz," IEEE Antennas and Wireless Propagation Letters, vol. 9, Article ID 5510104, pp. 682-685, 2010.

[14] S. S. Ghassemzadeh, R. Jana, C. W. Rice, W. Turin, and V. Tarokh, "A statistical path loss model for in-home UWB channels," in Proceedings of the IEEE Conference on Ultra Wideband Systems and Technologies, pp. 59-64, 2002.

[15] V. Erceg, L. J. Greenstein, S. Y. Tjandra et al., "Empirically based path loss model for wireless channels in suburban environments," IEEE Journal on Selected Areas in Communications, vol. 17, no. 7, pp. 1205-1211, 1999. 

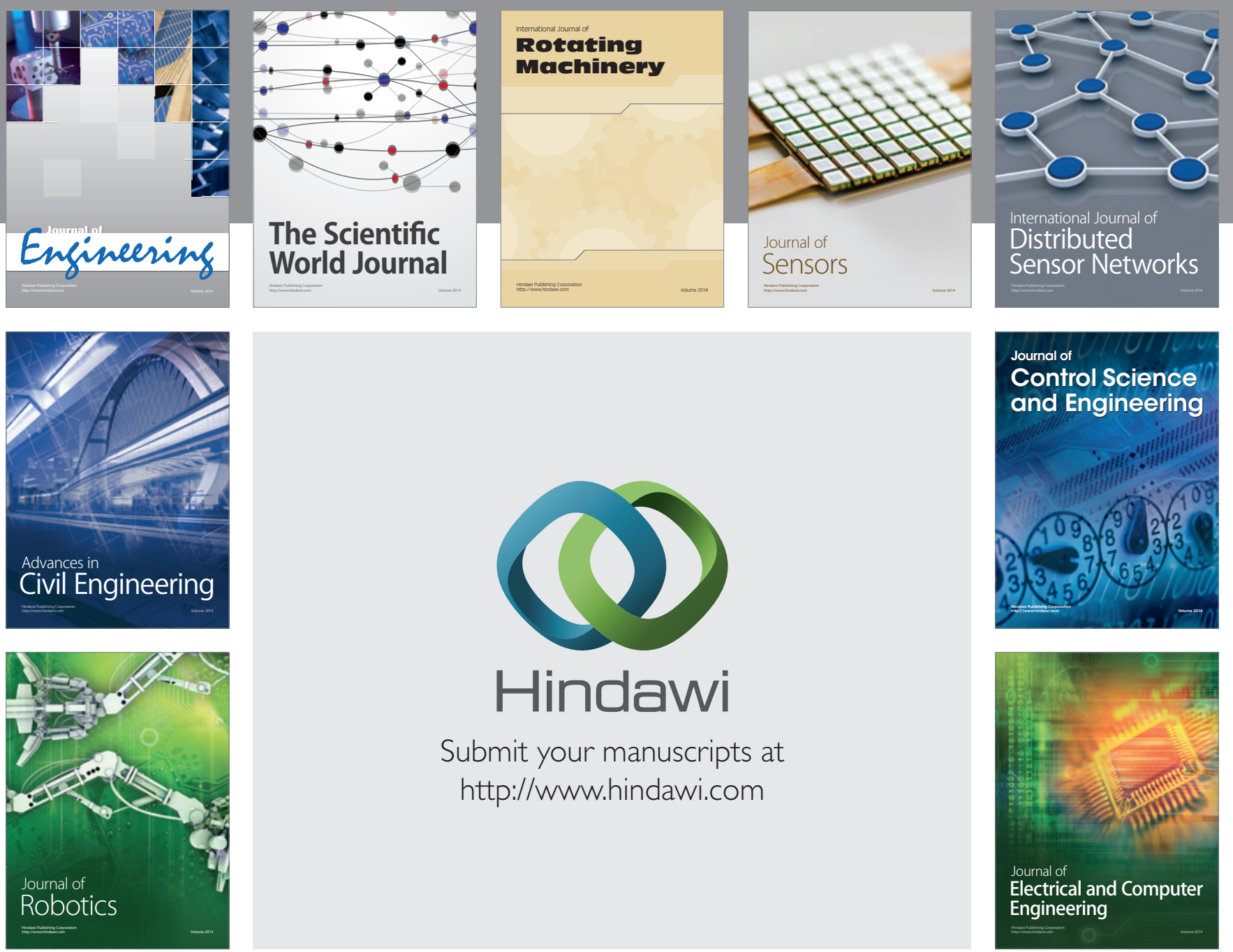

Submit your manuscripts at

http://www.hindawi.com
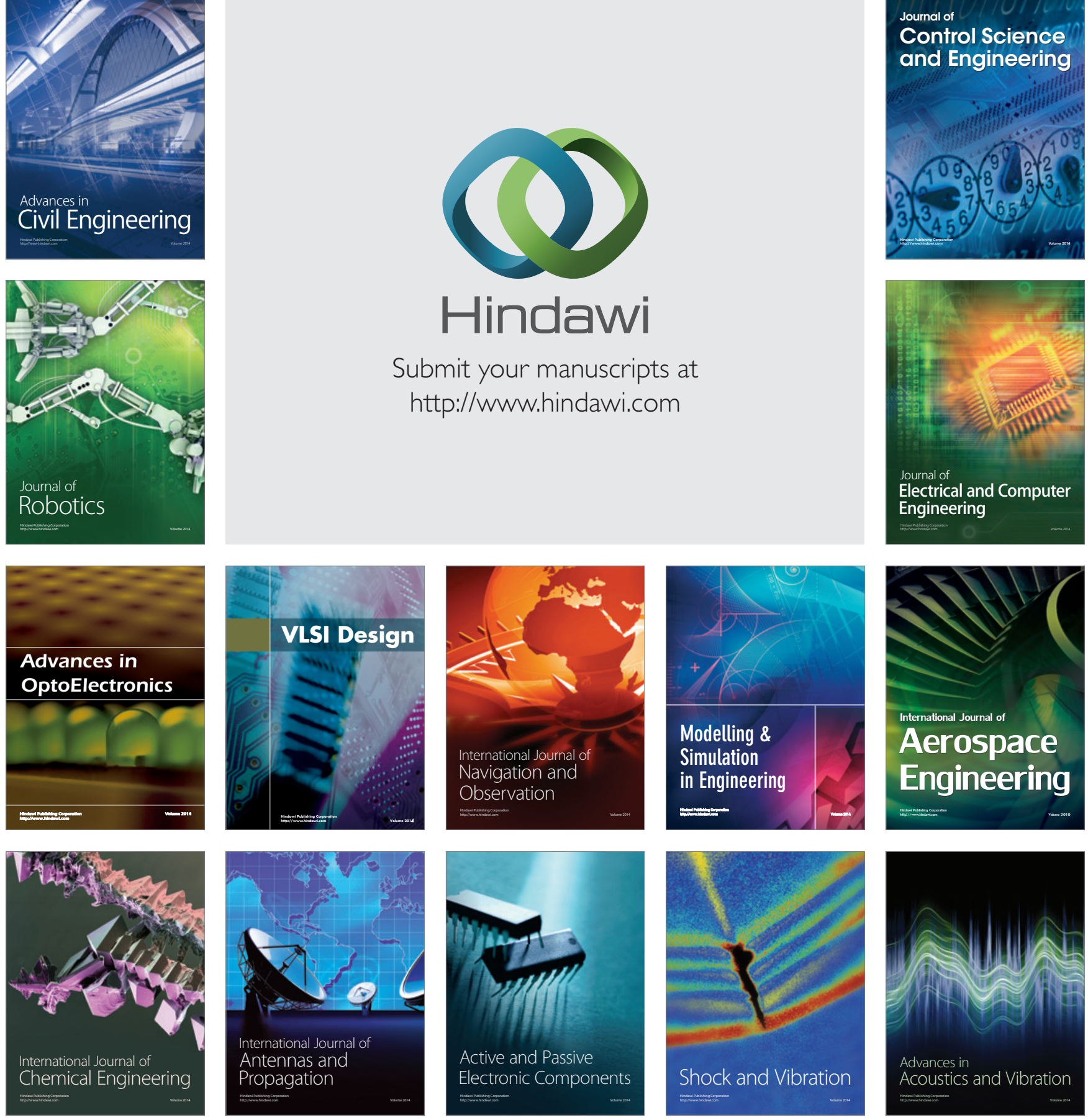